Signal \& Image Processing : An International Journal (SIPIJ) Vol.3, No.2, April 2012

\title{
AN APPROACH TO FACE RECOGNITION OF 2-D IMAGES USING EIGEN FACES AND PCA
}

\author{
Annapurna Mishra ${ }^{1}$, Monorama Swain ${ }^{2}$ and Bodhisattva Dash ${ }^{3}$ \\ Department of Electronics \& Telecommunication Engineering \\ Silicon Institute of Technology, Bhubaneswar, India \\ annapurnamishra12@gmail. $\mathrm{com}^{1}$, manoramamanu@gmail. $\mathrm{com}^{2}$, \\ budhisattavesilicon.ac.in ${ }^{3}$
}

\begin{abstract}
Face detection is to find any face in a given image. Face recognition is a two-dimension problem used for detecting faces. The information contained in a face can be analysed automatically by this system like identity, gender, expression, age, race and pose. Normally face detection is done for a single image but it can also be extended for video stream. As the face images are normally upright, they can be described by a small set of 2-D characteristics views. Here the face images are projected to a feature space or face space to encode the variation between the known face images. The projected feature space or the face space can be defined as 'eigenfaces' and can be formed by eigenvectors of the face image set. The above process can be used to recognize a new face in unsupervised manner. This paper introduces an algorithm which is used for effective face recognition. It takes into consideration not only the face extraction but also the mathematical calculations which enable us to bring the image into a simple and technical form. It can also be implemented in real-time using data acquisition hardware and software interface with the face recognition systems. Face recognition can be applied to various domains including security systems, personal identification, image and film processing and human computer interaction.
\end{abstract}

\section{KEYWORDS}

Face Recognition; Feature Space, Eigen Faces, Eigenvectors.

\section{INTRODUCTION}

One of the most recent biometric identification techniques is Face recognition, which can recognize an individual person based on their facial features as essential elements of distinction. Here we consider the problem as; for given still or video images of a particular scene, the identification of one or more persons in the scene using the technique from the stored database of faces. Correlation method is the first approach used for face recognition. But to reduce the computational expense, the dimensionality reduction processes are derived. The two important approaches for face recognition are: geometric (feature based) and photometric (view based).The three different algorithms developed by researchers are: Principal Components Analysis (PCA), Linear Discriminant Analysis (LDA), and Elastic Bunch Graph Matching (EBGM). 
Signal \& Image Processing : An International Journal (SIPIJ) Vol.3, No.2, April 2012

\section{A. PCA: Principal Components Analysis (PCA)}

The use of eigenfaces is commonly called as Principal Component Analysis (PCA).With PCA, the images must be used of the same size and they are normalized to line-up the eyes and mouth of the subjects within the images. The dimension of the data using data compression basics is reduced using PCA and that reveals the most effective low dimensional structure of facial patterns. Using this low dimension structure it precisely decomposes the face structure into orthogonal and uncorrelated components known as eigenfaces.Using this technique the face image can be represented as a weighted sum or feature vector of the eigenfaces which can be stored in a 1-D array. To avoid the poor performance of the result image, the PCA approach requires the full frontal face to be presented each time. This technique reduces the required data to identify the individual to $1 / 1000^{\text {th }}$ of the presented data.

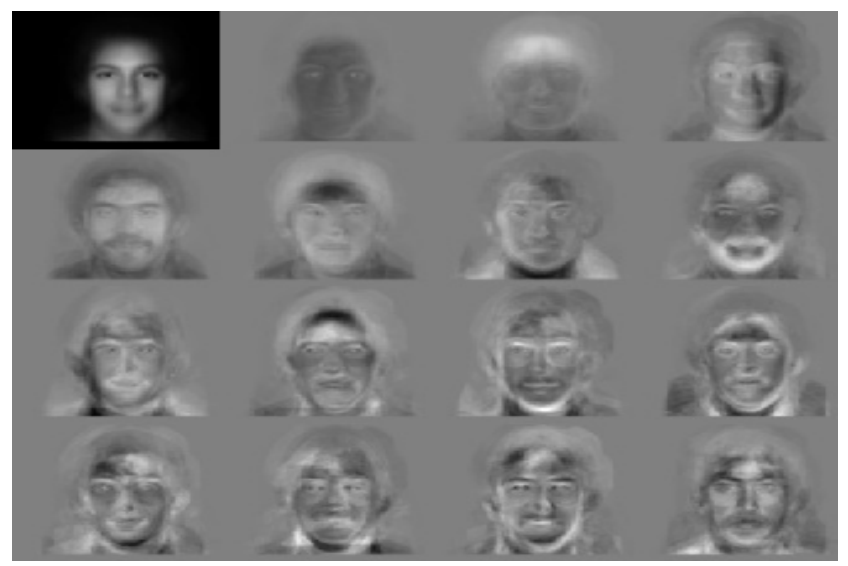

Figure 1: Standard Eigenface

\section{B. LDA: Linear Discriminant Analysis}

The statistical approach for classification of samples of unknown classes based on training samples with known class is called Linear Discriminant Analysis. This approach maximizes the variance across users called the between-class variance and minimizes the variance within classes called within class variance. In Figure 2 where each block represents a class, there are large variances between classes, but little variance within classes. When dealing with high dimensional face data, this technique faces the small sample size problem that arises where here are a small number of available training samples compared to the dimensionality of the sample space.

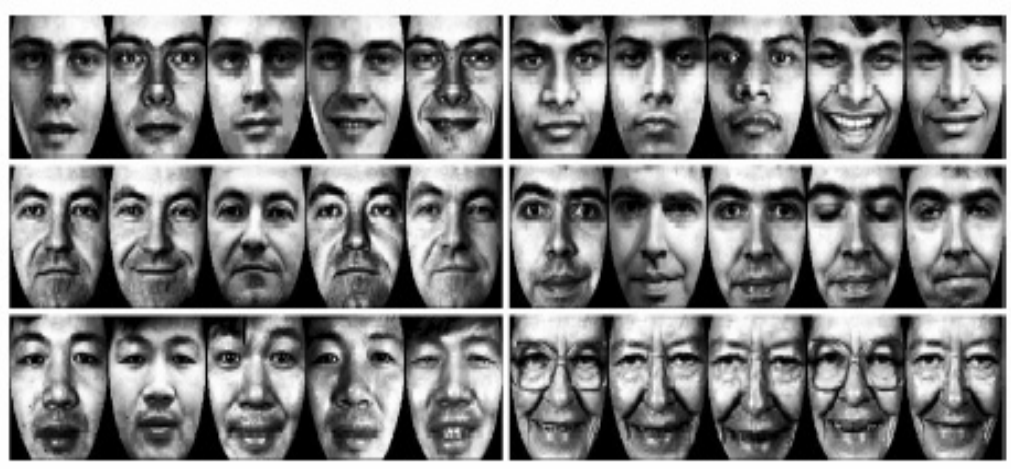

Figure 2 : Example of Six Classes Using LDA. 
Signal \& Image Processing : An International Journal (SIPIJ) Vol.3, No.2, April 2012

\section{EBGM: Elastic Bunch Graph Matching}

EBGM relies on the concept that real face images have many non-linear characteristics such as variations in illumination (outdoor lighting vs. indoor fluorescents), pose (standing straight vs. leaning over) and expression (smile vs. frown). A dynamic link architecture projecting the face onto an elastic grid can be formed using a Gabor wavelet transform. As shown in the image Figure 3, the Gabor jet forms a node on the elastic grid which describes the behaviour of the image around a given pixel. To detect the shapes and to extract the features of the image, the Gabor filter is convolved with the given image. Also the Gabor filter process can be used in biological-based methods, for example: in the visual cortex of higher mammals. Sometimes the limitations of this method can be achieved by combining PCA and LDA methods like accurate landmark localization.

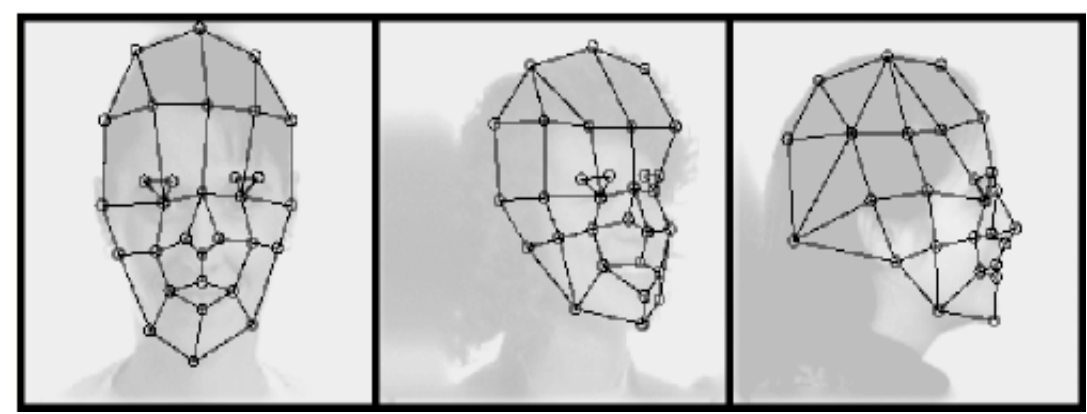

Figure 3 : Elastic Bunch Map Graphing.

\section{LITERATURE REVIEW}

1. Matthew A. Turk and Alex P. PentlandVision and Modeling Group, The Media Laboratory Massachusetts Institute of Technology, in their paper "Face Recognition using Eigen Faces" described about an overview of the detection and identification of human faces. They also described a working, near-real-time face recognition system which tracks a subject's head and then recognizes the person by comparing characteristics of the face to those of known individuals.

2. Nilima B Kachare and Vandana S Inamdar in their journal 'Survey of Face Recognition Techniques'. International Journal of Computer Applications have discussed about past research on biometric face feature extraction and recognition of static images. They explained about the implementation outline of these methods along with their comparative measures.

\section{PROPOSED METHOD FOR FACE RECOGNITION}

Here if we go through the literature [21,22] two main approaches to feature extraction have been extensively used. The first one is based on extracting structural facial features that are local structures of face images, for example; the shapes of the eyes, nose and mouth. The structure based approaches are not affected by irrelevant data, such as hair or background because they deal with local data instead of global data. Again, the statistical based approaches extract features from the whole image. From the psychological viewpoint, Hay and Young [23] pointed out that the internal facial features such as the eyes, nose and mouth are very important for human beings to see and to recognize familiar faces. 
Signal \& Image Processing : An International Journal (SIPIJ) Vol.3, No.2, April 2012

The proposed problem consists of verifying if a new face image belongs to one of the individuals, whose images were stored in a database in a similar way, aiming the person's identity in case of recognition $[1,15]$.A new face is compared with well-known faces stored in a database, being classified as a well known individual's face or as an unknown face [16]. Face representation techniques, that can be framed in three different categories: Template-based, Feature-based and Appearance-based $[2,17,19]$. Template-based method represents the faces by means of a main two-dimensional template with values representing the facial ellipse borders and of all face organs. Feature-based method considers the positions and sizes of the facial organs, such as eyes, nose, mouth, etc., in the face representation. This method consumes very less computer resources than the template-based method. Appearance-based method intends to project the face images in a low dimension subspace, to obtain the face representation. The Eigen faces space is an application of this method. It is built on Principal Component Analysis, from the projection of the images of the training set into the face space with low dimension. The Eigen faces space is an application of this method. It is built on Principal Component Analysis, from the projection of the images of the training set into the face space with low dimension. The objective of the Principal Component Analysis is to take the total variation on the training set of faces and to represent this variation with just some little variables. PCA intends to reduce the dimension of a group or to space it better so that the new base describes the typical model of the group [3]. The eigenfaces are the principal components of the original face images, obtained by the decomposition of PCA, forming the face space from these images [11]. So any new face can be expressed as linear combination of these Eigen faces. Face recognition is performed from the projection of the analyzed face into the face space and the measuring of the Euclidean distance between the new face and the face classes. The face space is described by an eigenfaces group. Each face is represented by its projection on the face space expanded by the eigenfaces [9, 14].Areas such as network security, content indexing and retrieval and video compression benefits from face recognition technology because 'people' are the center of attention in a lot of video. Face recognition is one of the few biometric methods that possess the merits of both high accuracy and low intrusiveness.

\section{a) Eigenfaces Approach}

Eigen space-based approaches approximate the face images with lower dimensional feature vectors. To produce the low dimension feature vectors let us consider that the face space given by the feature vectors has a lower dimension than the image space represented by the number of pixels in the image. Again the face recognition can be performed in this reduced space. This complete procedure considering the training to create the face database, the projection matrix to achieve the dimensional reduction is obtained from the database of face images $[10,13]$.

\section{b) Proposed Algorithm}

- Form the Training Set, collecting the initial set of face images.

- Define the face space of $\mathrm{M}$ images by calculating eigenfaces from the training set keeping only $\mathrm{M}$ images corresponding to the highest eigenvalues

- Calculate distribution in this M-dimensional space for each known person by projecting their face images onto this face-space. Projecting this new image onto each of the eigenfaces, calculate a set of weights based on $\mathrm{M}$ eigenfaces for a given input image. 
Signal \& Image Processing : An International Journal (SIPIJ) Vol.3, No.2, April 2012

- Determine whether the image is face or not by checking if the image is sufficiently close to face-space.

- For faceimage, the weight pattern is classified as either known or unknown person.

- The weight pattern can be compared with known weight patterns to match faces.

A face recognition algorithm can be divided into the following functional modules: a face image detector that finds the locations of human faces from a normal picture against simple or complex background and a face recognizer which determines" who this person is". Both the face detector and face recognizer follow the same framework, they both have a feature extractor that transforms the pixels of the facial image into a useful vector representation and a pattern recognizer that searches the database to find the best match to the incoming face image.

\section{c) Face Recognition from Eigenfaces}

The previous research work on automated face recognition shows that some aspects of the face stimulus are also important for face recognition. This suggests the use of an information theory approach of coding and decoding of face images, emphasizing the significant local and global features. The features such as the eyes,nose,lips and hair may or may not be directly related to the local and global features of the face image. In the language of information theory, the relevant information in a face image is extracted, encoded as efficiently as possible, and then compared with a database of models encoded similarly. To extract the information contained in a face image is the variation in a collection of face images that is captured, independent of any features and this information is used to encode and compare individual face images. The Eigen vectors are ordered, each one accounting for a different amount of the variation among the face images. A simple approach to extracting the information contained in an image of a face is to somehow capture the variation in a collection of face images, independent of any judgment of features, and use this information to encode and compare individual face images. The Eigen vectors are ordered, each one accounting for a different amount of the variation among the face images. These eigenvectors can be thought of as a set of features that together characterize the variation between face images. Each image location contributes more or less to each eigenvector, so that it is possible to display these eigenvectors as a sort of ghostly face image which is called an "eigenface".

When a new face image is given as input to check for face recognition, then it can be classified in one of the defined image classes [7]. Also it can be compared for match with any of the existing images in database. Face images are represented by intensity values of each pixel. So for example, if the images have dimension $256 \times 256$ pixels, then the dimension of the image vector will be 65536 . So here $\mathrm{N}=65536$. Normally for all images, since dimensionality of image is large, the value of $\mathrm{N}$, dimension of image vector is also large. Say new image is $\Gamma$. This can be

represented as column vector of dimension $\mathrm{Nx} 1$. This new image is mean centered by subtracting average face $\Psi$.

Each of such new face submitted to the Face Recognition is projected into the face space, obtaining the vector $\Omega$, also known as Face Key for this image, by using equation (1).

$$
\Omega=U^{T}(\Gamma-\Psi)
$$


Signal \& Image Processing : An International Journal (SIPIJ) Vol.3, No.2, April 2012

This vector with dimension (Mx1) is compared with each vector $\Omega$ i representing face keys for each of class images. If the distance found among $\Omega$ and any $\Omega \mathrm{i}$ is inside threshold of the class and is the smallest found distance, then there is a facial recognition of $\Omega$ belonging to class image i. This Euclidean distance between two face key vectors can be calculated using square minimal method. Face images are represented by intensity values of each pixel. Let the dimensionality of each image be $\mathrm{m} \times \mathrm{n}$. This means that each image consists of grid of pixels with $\mathrm{m}$ rows and $\mathrm{n}$ columns. Let $\mathrm{I}(\mathrm{x}, \mathrm{y})$ represents intensity values for all pixels. Since dimensionality of image is large, the value of $\mathrm{N}$, dimension of image vector is also large. This maps the image to collection of points in huge space. As the faces are similar in overall configuration, these images will not be randomly distributed in huge space and thus will lie in relatively low dimensional space.

Let the training set consists of $\mathrm{M}$ images representing $\mathrm{M}$ image classes. Each of these images can be represented in vector form as stated above. The average face of the set is given by equation (2)

$$
\Psi=\frac{1}{M} \sum_{i=1}^{M} \Gamma_{i}
$$

Each face image defers from the average face of the distribution, and this distance is calculated by subtracting the average face from each face image. This gives us new image space as given in equation (3).

$$
\Phi_{i}=\Gamma_{i}-\Psi(i=1, \ldots, M)
$$

From this new image space of $\mathrm{M}$, images (Each with dimension $\mathrm{N} \times 1$ ), the matrix $\mathrm{A}$ is formed with dimension $\mathrm{N} \times \mathrm{M}$ by taking each of image vectors and placing them in each column of matrix A given by equation (4)

$$
A=\left[\phi_{1} \ldots \phi_{M}\right]
$$

Using matrix $\mathrm{A}$, it is important to set up the Covariance matrix $\mathrm{C}$. This can be given by product of matrix A with matrix $A^{T}$. The dimension of such covariance matrix will be $\mathrm{N}$ x $\mathrm{N}$ given by equation (5)

$$
C=A A^{T}
$$

As the dimension of this matrix is $\mathrm{N} \mathrm{x} \mathrm{N}$, which means it will result in $\mathrm{N}$ Eigen values and $\mathrm{N}$ eigenvectors. Since the value of $\mathrm{N}$ is very large, say 65536 as in above example, it would be better to reduce this overhead by considering matrix as given in equation(6). The dimension of this matrix will be $\mathrm{M}$ x M.

$$
L=A A^{T}
$$

The eigenvalues obtained from $\mathrm{C}$ are same as $\mathrm{M}$ eigenvalues with remaining $\mathrm{N}-\mathrm{M}$ eigenvalues equals to zero. Also if $\mathrm{x}$ is eigenvector obtained from $\mathrm{C}$ then the eigenvectors of $\mathrm{L}$ are given by equation (7),

$$
y=A^{T} x
$$


Signal \& Image Processing : An International Journal (SIPIJ) Vol.3, No.2, April 2012

This relationship is used to obtain eigenvalues and eigenvectors of $A A^{T}$ by calculating eigenvalues and eigenvectors for $A^{T} A$. The eigenvectors for $\mathrm{C}$ say matrix $\mathrm{U}$ are obtained from eigenvectors of L say matrix $\mathrm{V}$ in equation (8),

$$
\mathrm{U}=\mathrm{AV}
$$

The matrix $\mathrm{V}$, with dimension $(\mathrm{M} \times \mathrm{M})$, is constituted by the $\mathrm{M}$ eigenvectors of $\mathrm{L}$ and matrix $\mathrm{U}$, with dimension ( $\mathrm{N} \mathrm{x} \mathrm{M}$ ), is constituted by all the eigenvectors of $\mathrm{C}$, and the matrix $\mathrm{A}$ is the image space, with dimension ( $\mathrm{N} \times \mathrm{M})$. The eigenfaces can be simply defined as the Eigenvectors which represent one of the dimensions of face image space [7]. The eigenfaces are a group of important characteristics that describe the variation in the group of face images. All the images from training set are projected to this eigenspace. These can be represented by linear combination of the eigenfaces that have a new descriptor as a point in a great dimensional space. This projection is constructed in equation (9)

$$
\Omega_{i}=U^{T}(\Gamma-\Psi) \text { where }(i=1, \ldots, M)
$$

As the projection on the eigenfaces space describes the variation of face distribution, it is possible to use these new face descriptors to classify them [7].

\section{d) Initialization for Input Image}

When a new face image is given as input to check for face recognition, then it can be classified in one of these image classes. Also it can be compared for match with any of the existing images in database. Say new image is $\Gamma$. This can be represented as column vector of dimension $\mathrm{Nx} 1$. This new image is mean centered by subtracting average face $\Psi$.

\section{e) Calculate Face Key Vector}

Each of such new face submitted to the Face Recognition is projected into the face space, obtaining the vector $\Omega$, also known as Face Key for this image, by using equation (10).

$$
\Omega=U^{T}(\Gamma-\Psi)
$$

\section{f) Classification of Input Image}

This vector with dimension (Mx1) is compared with each vector $\Omega_{i}$ representing face keys for each of class images. If the distance found among $\Omega$ and any $\Omega_{i}$ is inside threshold of the class and is the smallest found distance, then there is a facial recognition of $\Omega$ belonging to class image i. This Euclidean distance between two face key vectors can be calculated using square minimal method given by equation (11).

$$
\varepsilon_{i}^{2}=\left\|\Omega-\Omega_{i}^{2}\right\|
$$

\section{g) Face Recognition}

This image can also be checked for match with one of the existing images in the database. As the database will store the face keys for each of the images, by finding Euclidean distance between 
this new image face key vectors $\Omega_{a}$ with face key vector $\Omega_{b}$ which represents one of the images in database, the match can be checked. The proposal is to find one threshold for each class, looking for a better acting to face recognition $[17,18]$. The $\Theta_{i}$ are the thresholds which define the maximum allowed distance among the new face submitted to recognition and each class image. If the distance found between the new face and one of the classes is inside the class threshold, then face recognition is found. This threshold value can be calculated as in equation (12),

$$
\Theta_{i}=\frac{1}{k} \max \left(\Omega_{i}-\Omega_{j}\right)
$$

On this approach we use factor $\mathrm{k}$ from 1 to 10 . If this factor is little (near to 1), we have a big false-positive rate and a little false-negative rate [4]. Otherwise, if this factor is big (near to 10), we have a little false-positive rate and a big false-negative rate

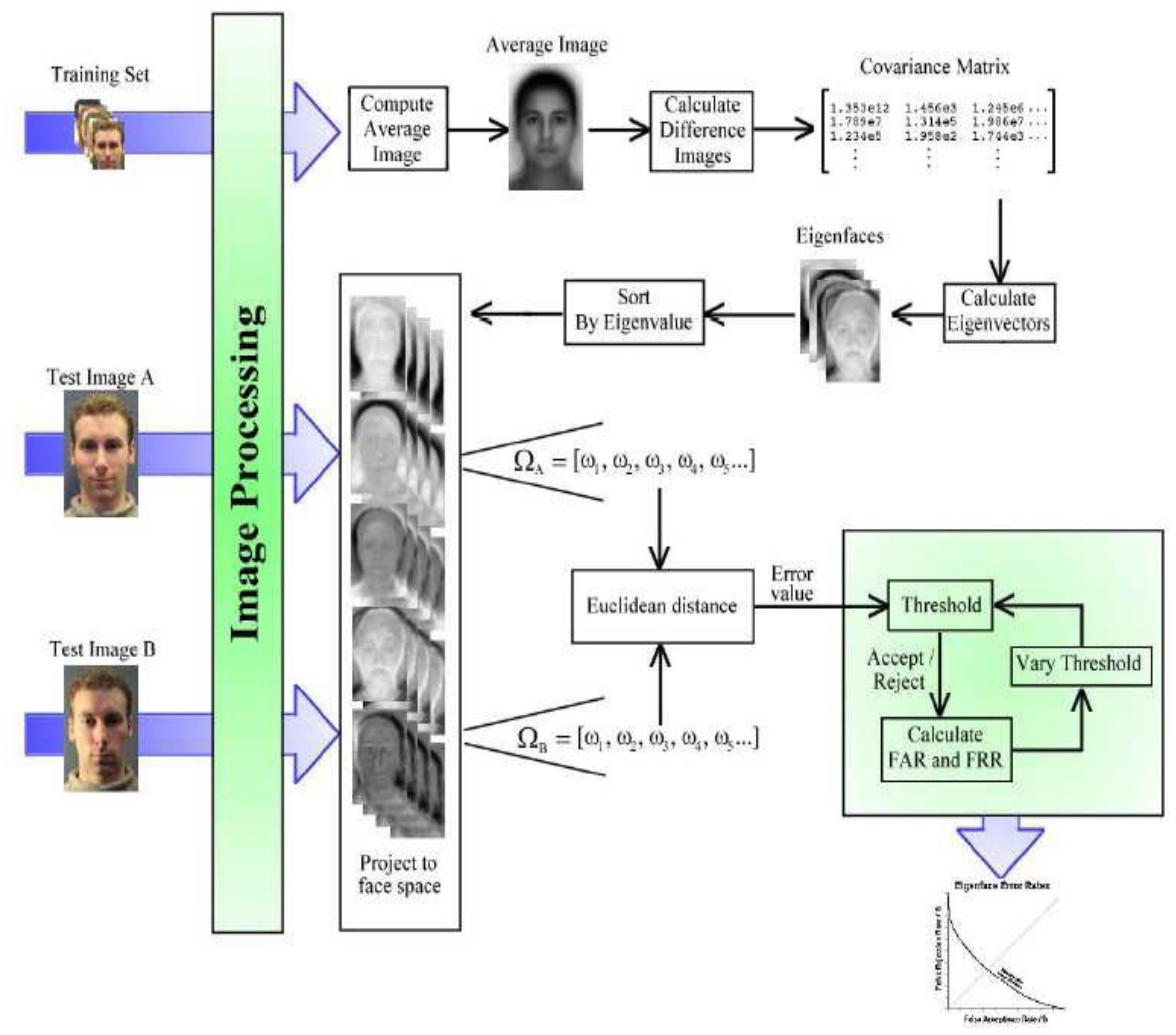

Figure4. Face Recognition Process

The training set of images are represented by the $\mathrm{M}$ eigenvectors [5].To represent face space spanned by images, only $\mathrm{M}^{\prime}$ Eigenvectors are chosen from $\mathrm{M}$ Eigenvectors, such that $\mathrm{M}^{\prime}$ is less than than M. Here the face space dimension is also reduced and enhance the speed for face recognition. So to reduce the dimension of face image M' Eigenvectors are chosen with highest Eigenvalues.As the higher Eigen values represent maximum face variation in the Eigenvector, so it is considered as the Eigenvector for face space representation [6,8]. Small Eigen values can be neglected to further reduce the dimension of face space as the lower Eigen values does not provide much information about face variations in corresponding Eigenvector direction [6, 13]. This does not affect the success rate much and is acceptable depending on the application of face recognition. Here only M' Eigenvectors with higher Eigenvalues are chosen for defining face 
Signal \& Image Processing : An International Journal (SIPIJ) Vol.3, No.2, April 2012

space $[1,2]$. The most important point here is to choose value of $\mathbf{M}^{\prime}$ so that it does not result in high error rates for Face recognition process [15]. The M' should be chosen such that error rates does not increase much and are acceptable depending on application for face recognition.

\section{RESULTS AND DISCUSSIONS}

The training set of images in fig.5 is given as input to find eigenspace. Using these images, the average face image is computed. The covariance matrix represents the difference of these images. This is used to calculate Eigenvectors and Eigen values in fig.8. These are the Eigen faces which represent various face features. So for given two test images are projected onto this eigenspace to give the weight vector also known as Face key for that image. The Euclidean distance between these two face key vectors is calculated [3]. If this is below some threshold value, then two images are said to be matching that means they belong to same person [10, 12, and 13]. Depending on this result, False Acceptation Rate (FAR) and False Rejection Rate (FRR) are found. An ID system with both low FAR and FRR is considered having good discriminating power. The two standard biometric measures to indicate the identifying power are FRR and FAR. The FRR-type 1 error and FAR-type 2 error are inversely proportional measurements, for example; if an ID system tunes its threshold value to reject all imposters or minimizing FAR ,it may also improperly reject some authorized users or maximizing FRR.Therefore ID system designers often provide a variable threshold setting for customers to strike a balance or to change value of threshold.

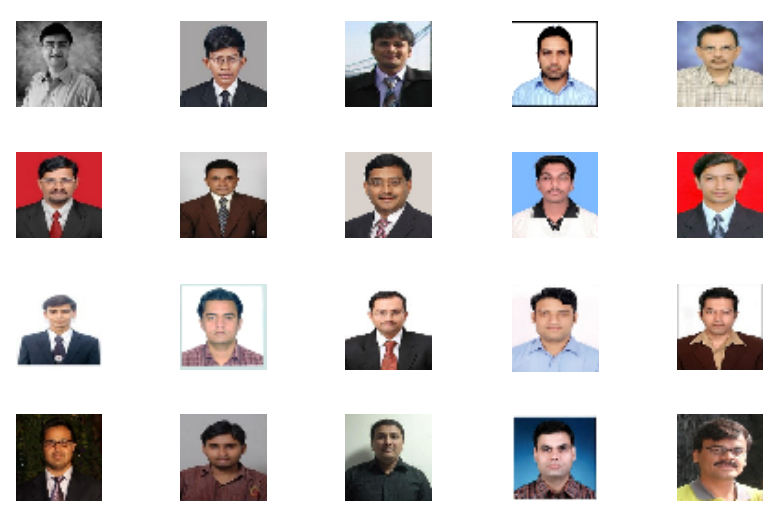

Figure5. Training Set. 
Signal \& Image Processing : An International Journal (SIPIJ) Vol.3, No.2, April 2012

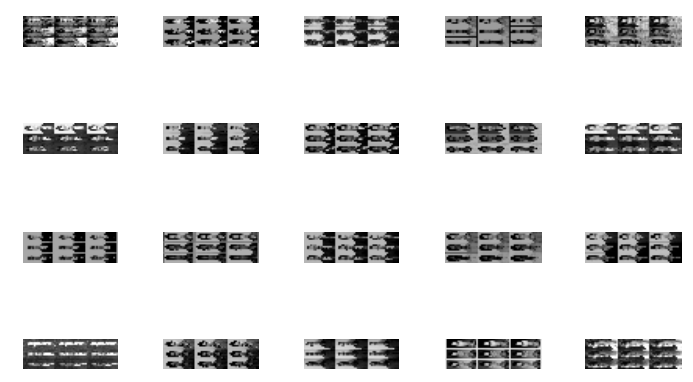

Figure6. Normalized Training Set.

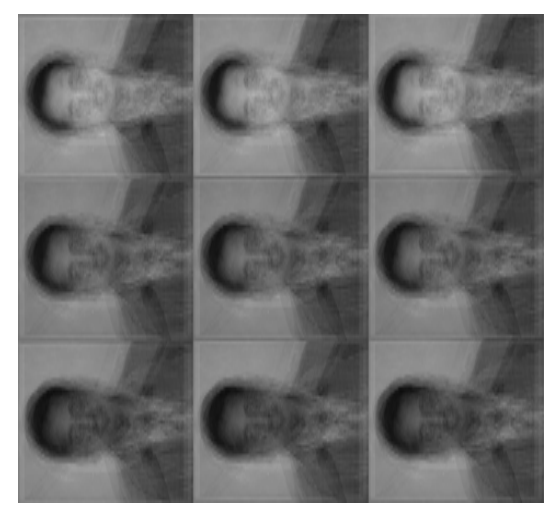

Figure7. Mean image

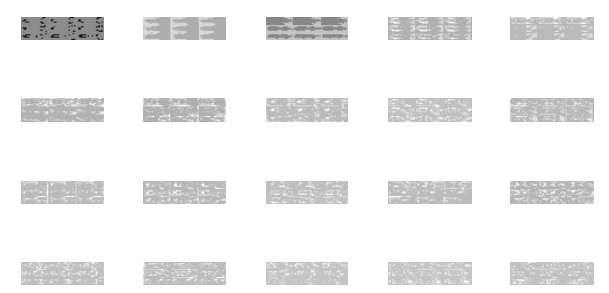

Figure8. Eigen faces of set of images 
Signal \& Image Processing : An International Journal (SIPIJ) Vol.3, No.2, April 2012
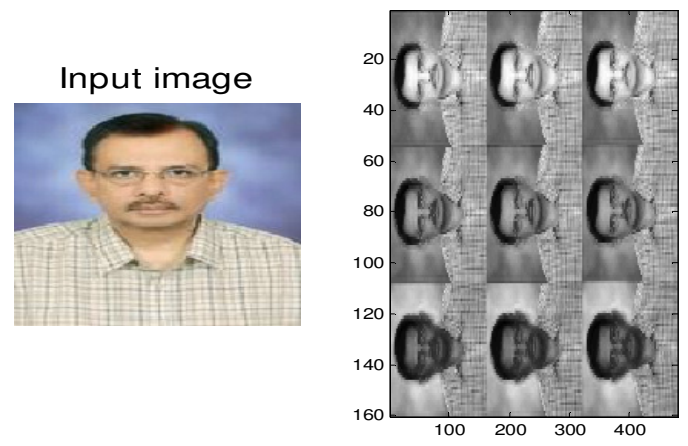

(a)
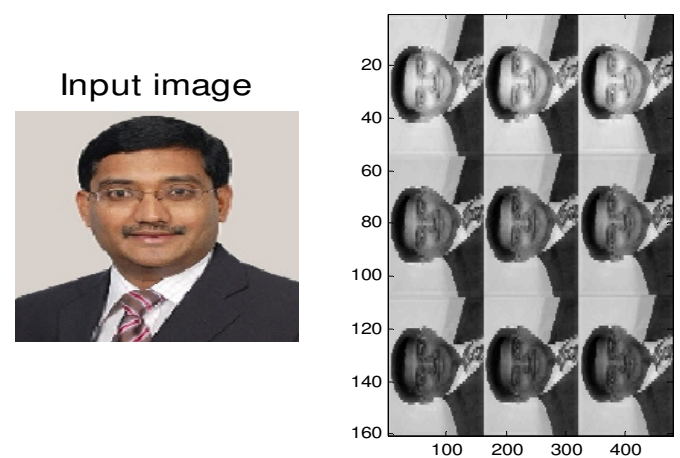

(b)

Figure9. Input test images (a), (b) and its corresponding reconstructed images
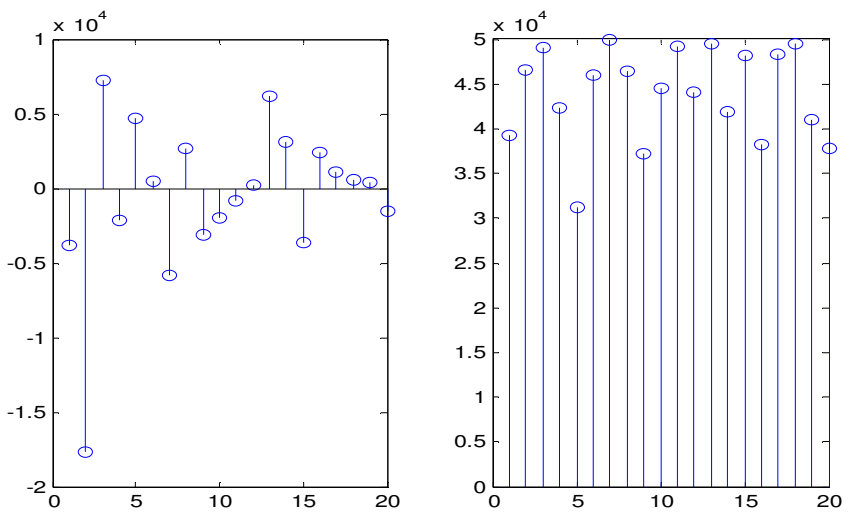

Figure10.Weight and Euclidian distance of input test images from figure 9(a). 
Signal \& Image Processing : An International Journal (SIPIJ) Vol.3, No.2, April 2012
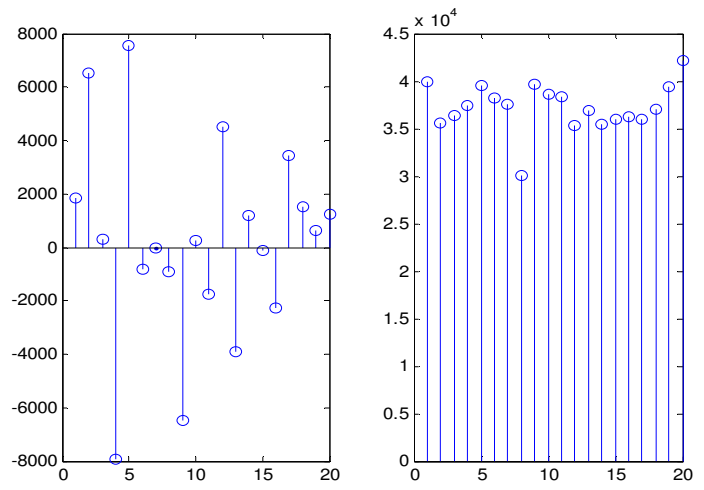

Figure11.Weight and Euclidian distance of input test images from figure 9(b).

\section{CONCLUSION}

Face recognition is a both challenging and important recognition technique. Among all the biometric techniques, face recognition approach possesses one great advantage, which is its userfriendliness or non-intrusiveness. In this paper, the initial survey face recognition technique is discussed. The Eigen face approach for Face Recognition process is fast and simple which works well under constrained environment. Here we have covered issues such as the generic framework for face recognition, factors that may affect the performance of the recognizer, and several stateof-the-art face recognition algorithms. The Eigen face approach for Face Recognition process is fast and simple which works well under constrained environment. So instead of searching large database of faces, it is better to give small set of likely matches. By using Eigen face approach, this small set of likely matches for given images can be easily obtained. By using eigenface approach, we try to reduce this dimensionality. The eigenfaces are the eigenvectors of covariance matrix representing the image space. The lower the dimensionality of this image space, the easier it would be for face recognition. Any new image can be expressed as linear combination of these eigenfaces. We have also seen that taking eigenvectors with higher $\mathrm{M}^{\mathrm{\prime}}$ eigenvalues instead of all $\mathrm{M}$ eigenvectors, does not affect performance much. So even taking lower dimensional eigenspace for the images is acceptable as error rates in such cases are very low. More research needs to be done on choosing the best value of $\mathrm{M}^{\prime}$. This value of $\mathrm{M}^{\prime}$ may vary depending on the application of Face Recognition. So various methods for making best choice of $\mathbf{M}^{\prime}$ needs to be studied [18, 20]. One of the limitations for eigenface approach is in the treatment of face images with varied facial expressions and with glasses. A real-time system can be implemented using suitable data acquisition software and hardware interface with digital face recognition systems.

\section{ACKNOWLEDGMENT}

The authors thank to Dr. R. N. Pal, retired Professor IIT Kharagpur, India and his team for their help in conducting experiments with different face images. The authors wish to thank Prof. Rutuparna Panda for his valuable suggestions that improved the quality of the paper. 
Signal \& Image Processing : An International Journal (SIPIJ) Vol.3, No.2, April 2012

\section{REFERENCES}

[1] J. Goldstein, L. D. Harmon, and A. B. Lesk, "Identification of Human Faces," Proc. IEEE, May 1971, Vol. 59, No. 5, 748-760.

[2] L. Sirovich and M. Kirby, "A Low-Dimensional Procedure for the Characterization of Human Faces," J. Optical Soc. Am. A, 1987, Vol. 4, No.3, 519-524.

[3] J. Lu, K.N. Plataniotis, and A.N. Venetsanopoulos, "Regularized Discriminant Analysis For the Small Sample Size Problem in Face Recognition," Pattern Recognition Letters, December 2003, Vol. 24, Issue 16: 3079-3087.

[4] P. J. Phillips, H. Moon, S. A. Rizvi, and P. J. Rauss, "The FERET Evaluation Methodology for FaceRecognition Algorithms," IEEE Transactions on PAMI, 2000, Vol. 22, No. 10: 1090-1104.

[5] P. J. Phillips, P. J. Flynn, T. Scruggs, K. W. Bowyer, J. Chang, K. Hoffman, J. Marques, J. Min, and W. Worek, "Overview of the Face Recognition Grand Challenge," Proc. Computer Vision and Pattern Recognition Conference, San Diego, 2005.

[6] Belhumeur, P., hespanha, J., and Kriegman, D., "Eigenfaces vs. Fisherfaces: Recognition Using Class Specific Linear Projection", IEEE Trans. Pattern Analysis Machine Intelligence, vol. 19, no. 7, pp.711-720, July 1997.

[7] Brunelli, R., and Poggio, T., "Face Recognition: Features versus Templates", IEEE Trans. Pattern Analysis Machine Intelligence, vol. 15, pp.1042-1052, 1993.

[8] Chan, Y., Lin, S.-H., and Kung, S.Y., "Video Indexing and Retrieval", Multimedia Technology for Applications, Sheu and Ismail editors.IEEE Press, 1998.

[9] W. Zhao, R. Chellappa, A. Krishnaswamy, "Discriminant Analysis of Principal Components for Face Recognition", Proc. of the 3rd IEEE International Conference on Face and Gesture Recognition, FG'98, 14-16 April 1998, Nara, Japan, pp. 336-341.

[10] J.R.Beveridge, K.She, B.Draper, G.H.Givens, "A nonparametric Statistical Comparison of Principal Component and Linear Discriminant Subspaces for Face Recognition",Proc.of the IEEE conference on Computer Vision and Pattern Recognition, December 2001,Kaui,HI,USA,pp.535-542.

[11] K.Baek, B.Draper, J .R.Beveridge, K.She,"PCA vs.ICA:A Comparison on the FERET Data Set", Proc.of the Fourth International Conference on Computer Vision, Pattern Recognition and Image Processing,Durham,NC,USA,8-14 March 2002,pp.824-827.

[12] C. Liu, H.Wechsler,"Comparative Assessment of Independent Component Analysis (ICA) for Face Recognition", Second International Conference on Audio-and Video based Biometric Person Authentication, Washington D.C., USA, 22-23 March 1999.

[13] D.Swets and J.Weng,"Using discriminant eigenfeatures for image retrieval”, IEEE Trans. On Pattern Analysis and Machine Intelligence, vol.18, no.8, pp.831-836, 1996.

[14] R.Chellappa, C. Wilson, and S. Sirohey,"Human and machine recognition of faces: A survey", Proceedings of the IEEE, vol.83, no.5, pp.705-740, 1995.

[15] G.Yang and T, S.Huang,"Human face detection in a complex background", Pattern Recognition, vol.27, no.1, pp.53-63, 1994.

[16] Dollar,P.,Tu,Z.,Tao,H.,Belongie,S.:Feature mining for image classification.In:Proceedings of IEEE Conference on Computer vision and Pattern Recognition.(2007). 
Signal \& Image Processing : An International Journal (SIPIJ) Vol.3, No.2, April 2012

[17] Huang, C., Ai, H., Li, Y., Lao, S: High-performance rotation invariant multiview face detection.IEEE Transactions on Pattern Analysis and Machine Intelligence 29(4)(2007)671-686.

[18] P.Sinha, B.Balas, Y.Ostrovsky, and R.Russel,"Face recognition by humans: Nineteen results all computer vision researchers should know about, "Proceedings of the IEEE, vol.94, no.11, pp.19481962, 2006.

[19] B.Heisele, T.serre, and T.Poggio,"A component-based framework for face detection and identification, "to appear in International Journal of Computer Vision, 2007.

[20] A.Hyvarinen,"Survey on independent component analysis, "Neural Computing Surveys, vol.12, pp.94-128, 1999.

[21] M.Turk and A Pentland,"Eigenfaces for recognition", Journal of Cognitive Neuroscience, vol.3, No.1, pp.71-86, 1991.

[22] "Theme section-face and gesture recognition", IEEE, Trans on Pattern Analysis and Machine Intelligence, vol.19, no.7, 1997.

[23] D.Hay and A.W.Young, Normality and Pathology in Cognitive Functions, Chapter the Human Face, Academic Press, 1982. 